\title{
A quantified study of segmentation techniques on synthetic geological XRM and FIB-SEM images
}

\author{
Matthew Andrew ${ }^{1}$ \\ Received: 2 March 2018 / Accepted: 15 August 2018 / Published online: 28 August 2018 \\ (C) The Author(s) 2018, corrected publication September/2018
}

\begin{abstract}
Three sets of synthetic images were created from two original datasets. A suite exhibiting greyscale contrast was produced from an $8.96-\mu \mathrm{m}$ voxel size 3D X-ray microscopy image of a sandstone rock and a two suites (one showing greyscale contrast and one showing both greyscale and textural contrast) were produced from a $5 \times 5 \times 5 \mathrm{~nm}$ voxel size FIB-SEM image of a shale rock. The performance of three image segmentation algorithms (global multi-Otsu thresholding, seeded watershed region growing, and machine learning-based multivariant classification) was then assessed by their ability to recover their respective original segmented 3D images. While all algorithms performed well at low noise levels, machine learning-based classification proved significantly more noise tolerant than either of the traditional algorithms. It was also able to segment the non-greyscale (textural based) contrast, something the traditional completely failed to do, with voxel misclassification rates for the traditional techniques above $50 \%$ at a 0 noise level within the textural contrast regions. Machine learning-based classification, in contrast, achieved misclassification rates of less than 5\% in the same regions.
\end{abstract}

Keywords Image processing $\cdot$ Image segmentation $\cdot$ Digital rock physics

\section{Introduction}

Digital rock analysis, a field which has developed from a primarily academic pursuit to an increasingly used industrial tool, is interested in the processes and mechanisms behind single and multiphase flow. In these analyses, properties of hydrogeological interest (e.g., porosity, absolute permeability, and the relative permeability and capillary pressure saturation functions) are computed computationally from discreet descriptions of the pore structure of the rock [1-3], generated using high-resolution microscopy techniques [4]. Recent advances have even allowed for the in situ measurement of fluid distributions [5-7], reactive transport $[8,9]$, and basic petrophysical properties such as local capillary pressures $[10,11]$ and contact angle [12-15]. These properties are then used to inform and populate reservoir simulation models, which in turn inform petroleum reservoir management decisions. They are, however, intimately related to the local pore microstructure, and as

Matthew Andrew

matthew.andrew@zeiss.com

1 Carl Zeiss Microscopy, 4385 Hopyard Rd, Pleasanton, CA, USA such, errors in segmentation result in errors in all subsequent analyses. Recent benchmarking efforts have shown that traditional segmentation techniques have the potential of inducing significant errors, particularly in flow and electrical transport parameters [16-18]. This is because, frequently, raw greyscale datasets contain both a variety of modality-specific artifacts and noise that cause, as resulting images become more complex, the failure of traditional segmentation approaches [19]. When visually inspecting such images, the brain acts to smooth out this noise and recognize patterns in the data to extract information through the artefacts, but such a process has frequently proved hard to automate and capture in a computational form. Traditionally, science has relied on the hard work of researchers to manually segment such artefact-ridden images. As datasets grow larger, more complex, and more multidimensional, however, these manual approaches become more and more 34 challenging to scale.

The last 20 years has seen a transformation in a wide range of fields, widely grouped together under the umbrella of "machine learning." While these technologies have transformed many areas of data science ranging from medical diagnosis to stock market analysis, image analysis for microscopy (outside some specific areas of application) has lagged behind developments in other fields. The power of 
such algorithms when applied to segmentation and classification problems in microscopy lies in their ability to create arbitrary classifiers which operate in much higherdimensional space than simply the image output from a specific microscope detector. These higher-dimensional spaces may be (spatially and/or temporally correlated) images acquired in different imaging modalities (i.e., using different detectors, energies, or techniques to extract different properties from the sample) or images derived from the original dataset by applying a range of filters to the sample (e.g., gradient, smoothing, or textural filters to extract different local and non-local features from the image). These features are then concatenated to from a single "feature vector," a set of numbers corresponding to all the (local, nonlocal, and textural) information created about that pixel. These techniques have begun to be applied to the segmentation of microscopic images, with recent studies on rock and soil samples including Chauhan et al. [20], Koebernick et al. [21], and Schweizer et al. [22].

In this paper, we perform quantitative benchmarking of classifiers created using machine learning algorithms against traditional image processing workflows across a range of samples of interest in the petroleum geosciences. One of the challenges in digital rock analysis is the multitude of different rock types of interest for analysis, requiring a multitude of imaging techniques to fully characterize. These range from high-porosity, high-permeability conventional resources (such as Siliciclastic sandstones [23, 24]) which have pore structures on the scale of tens of microns, through to unconventional shale resources with pore structures on the nanometer scale [25-29]. To examine the impact of differing segmentation techniques, three suites of 3D synthetic datasets were constructed from segmented real datasets of key samples, specifically high-resolution nanoscale focused ion beam-scanning electron microscopy (FIB-SEM) dataset (with a voxel size of $2.5 \times 2.5 \times 5 \mathrm{~nm}$, binned to a voxel size of $5 \times 5 \times 5 \mathrm{~nm}$ ) of an economic shale sample and a micron-scale X-ray microscopy (XRM, also known as micro-computed tomography or micro-CT) dataset (with a voxel size of $8.96 \times 8.96 \times 8.96 \mu \mathrm{m}$ ) of a model sandstone.

\section{Synthetic image reconstruction}

The two rock types analyzed in this study were Bentheimer Sandstone, an early Cretaceous high-porosity, highpermeability Quartz Arenite [30], and the Vaca Muerta shale, a late Triassic-early Jurassic economic low-tointermediate maturity onshore Argentinian shale. For the sandstone sample, the central portion of a micro-plug $10 \mathrm{~mm}$ in diameter and $50 \mathrm{~mm}$ in length was imaged using a ZEISS Xradia 520 Versa X-ray microscope or XRM
(ZEISS X-ray Microscopy, Pleasanton, CA). 3D volumes with a voxel size of $8.96 \mu \mathrm{m}$ were reconstructed using a proprietary filtered back projection algorithm [31] from a series of 1600 projections. Each projection consisted of $1024 \times 1024$ pixels and was reconstructed into a 3D volume of size $1024 \times 1024 \times 1024$ voxels, representing a physical volume of $9.14 \mathrm{~mm} \times 9.14 \mathrm{~mm} \times 9.14 \mathrm{~mm}$. This dataset was then segmented into two phases (pore and grain) using ZEISS Zen Intellesis [32], a graphical interface allowing for interactive training of machine learning classifiers from microscopy datasets. It uses open-source packages for both feature extraction (scikit-image (www.scikit-image. org) and SciPy (www.scipy.org)), multivariant classifier construction, simplification and application (scikit-learn (www.scikit-learn.org)), and task scheduling (Dask (https:// dask.pydata.org/en/latest/)). The interface allows for a direct and interactive "painting" of training data onto an image. Thirty-three features are then extracted from each greyscale channel, including 20 Gaussian filters (with standard deviations ranging from 0.7 to 8 pixels), five mean filters (of similarly varying kernel size), four Gabor filters (with two values for standard deviation (1 and 3) and two wavelengths (5 and 10 pixels)), one Hessian filter (giving three feature vectors corresponding to the $x x, x y$, and $y y$ second-order partial derivatives), and one Sobel feature vector. A multivariant classifier is then trained on these features using a "forest of random decision trees" approach [33]. This classifier is then applied to either the current 2D slice displayed for a 3D stack or a local region in a large 2D image. The user then iteratively adds or removes training data on multiple $2 \mathrm{D}$ slices through the $3 \mathrm{D}$ volume until a visually satisfactory segmentation is found. Once a desirable model has been trained, it can be applied across the entire 3D dataset, producing the final image segmentation (Fig. 1a, b).

This segmentation was then scaled such that the average greyscale value of each phase (pore and void) matched the average greyscale of that phase in the original XRM image. This ensured that resulting synthetic datasets had a greyscale value range representative of the original image, thereby maximizing the similarity of the resulting synthetic datasets to the original data. This volume was then forward-projected back into the projection domain, and a Gaussian blur (with a standard deviation of 1 pixel) was applied to the projection data (Fig. 1c). This data was represented as a dimensionless "absorption factor," ranging from 0 (where $0 \%$ of X-rays incident on the detector had been absorbed during $\mathrm{X}$ ray projection) to 1 (where $100 \%$ of $\mathrm{X}$-rays incident on the detector had been absorbed during X-ray projection). Gaussian noise with differing standard deviations was then applied to the projection data, creating a suite of synthetic projection datasets with noise levels ranging over an order of magnitude from 0.01 (representing a 

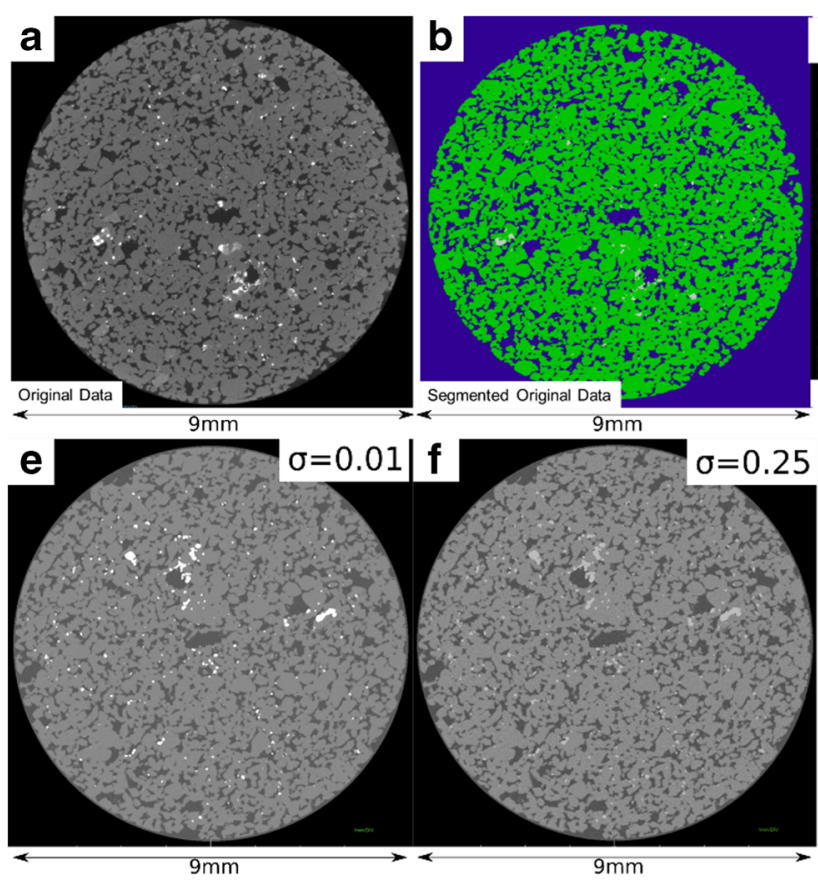

Fig. 1 The process for the generation of synthetic XRM data. a The original XRM data, showing pore (darkest phase) and grains (lighter phases). This was then segmented (b). The segmented data was then rescaled and re-projected (c), where a Gaussian blur and variable levels
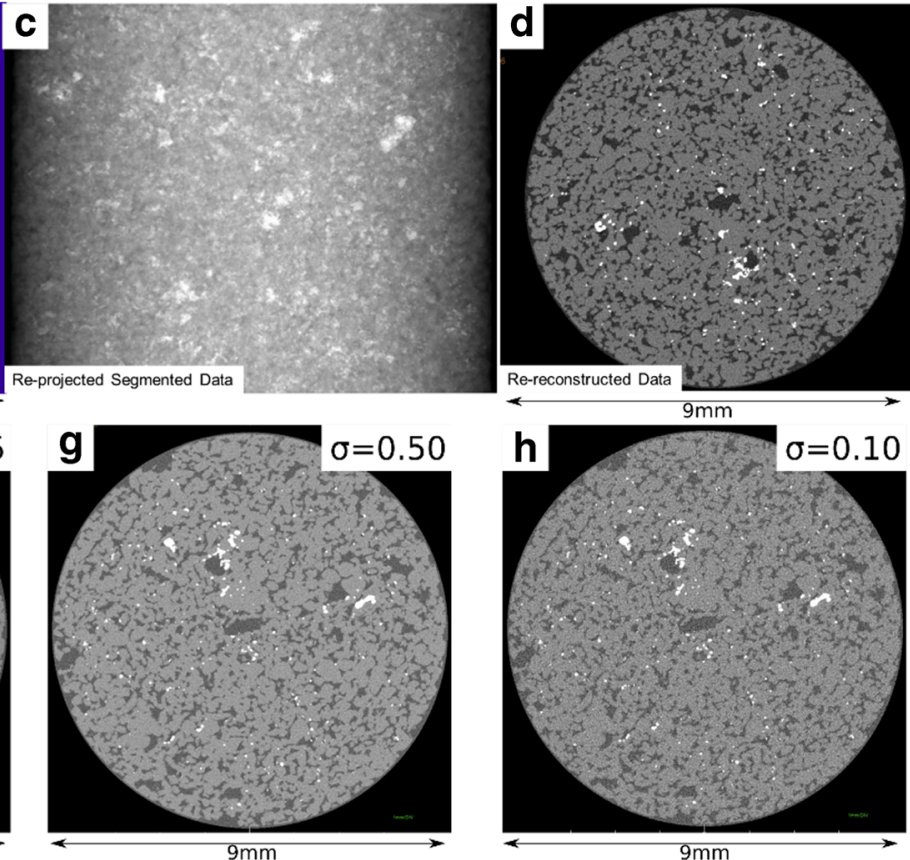

of noise were applied to the datasets before the suite of synthetic data was then reconstructed and variable noise levels $(\mathbf{d}-\mathbf{h})$. In each dataset $(\mathbf{e}-\mathbf{h})$, the noise level $(\sigma)$ is shown in the top right of the cross-sectional slice

learning into five phases (porosity, organic phases, quartz, calcite, and pyrite, in the order of their apparent greyscale value) to provide an initial segmentation baseline to which subsequent segmentations were then compared. One challenge when imaging porous materials using FIB-SEM is that of "pore backs" - an artefact where out-of-plane information is visible, interfering with segmentation of that slice. To simulate this in the synthetic data, a 1D convolutional ramp kernel was applied in the $Z$ (image slice) direction, effectively blurring the image in one direction (towards the front of the image). This kernel was only applied through the pore phase (out-of-plane information is only visible if it can be seen through the porosity) and had the effect of making nearby pore walls visible in plane (Fig. 2c, d). After this, 2D Gaussian blur (with a kernel size of 1 voxel) was applied. Two suites of data were then created. To create the first, the dataset was rescaled such that the average of each phase was equal to the average of that phase in the original FIB-SEM dataset, after which variable levels of Gaussian noise were added (with standard deviations ranging from 0 to 30 greyscale units) (Fig. 2e). This recreated the segmentation challenge faced with the XRM data, but with a greater number of desired phases for the final segmentation (five in the case of the FIB-SEM data, rather than two in the case of the XRM data). The second suite of synthetic FIB-SEM data constituted a qualitatively different (and more difficult to 

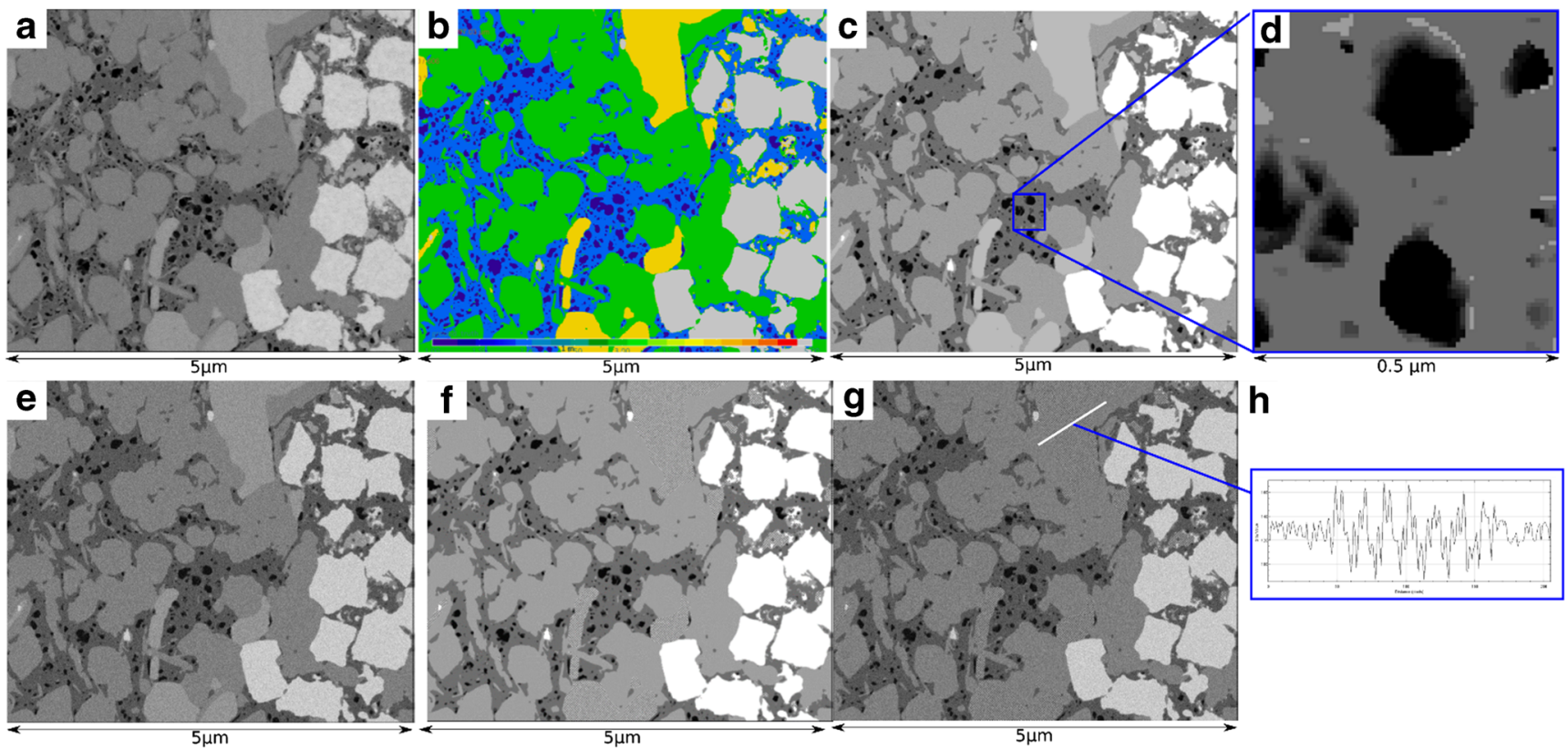

Fig. 2 The creation of a suite of synthetic FIB-SEM data from an original (real) dataset with a voxel size of $2.5 \times 2.5 \times 5 \mathrm{~nm}(\mathbf{a})$. b A five-phase segmentation of the dataset into (in order of greyscale intensity) pore, organic, quartz, calcite, and pyrite. $\mathbf{c}$ This segmentation was then rescaled back such that the average greyscale value of each pixel was equal to the average greyscale value of the associated phase in the original image. d "Pore Back" artefacts were introduced. e Variable levels of Gaussian noise were then applied to create a suite of "greyscale contrast" datasets. f "Textural contrast" datasets were created by applying a bidirectional sinusoid function through the "calcite" phase before adding Gaussian noise (g) solve) segmentation challenge-that of coupled greyscale and textural segmentation.

Frequently, the features of interest (such as different minerals or local microfacies) do not exhibit significant greyscale contrast. They may, however, display differences in texture or morphology [34], which are challenging to incorporate into a traditional image processing and analysis workflow (particularly when the segmentation is performed in parallel with segmentation relying on greyscale contrast). Machine learning presents a particularly powerful tool for segmentation using these parameters, so to simulate the impact of variations in texture in the synthetic data, the data was rescaled such that the average of each phase was equal to the average of that phase in the original FIB-SEM dataset apart from the second brightest phase (corresponding to the calcite mineral). This phase was given a greyscale equal to the average greyscale of the third brightest phase (corresponding to the quartz mineral) - there was therefore no difference in the average greyscale value of the regions corresponding to the quartz and the calcite in the "textural contrast" synthetic image. In order to differentiate these two phases, textural contrast was added to the "calcite" region by modulating the greyscale intensity of that region by a randomly modulating bidirectional sinusoidal function with a wavelength of 6 pixels. Varying levels of Gaussian noise were then applied to the resulting dataset, similar to the purely "greyscale contrast" dataset (and the suite of synthetic XRM datasets). The greyscale of the "calcite" region was therefore given by

$I_{\text {calcite, synthetic }}=I_{\mathrm{qz}}+A \times \sin X / \lambda+B \times \sin Y / \lambda+$ Noise $_{\mathrm{Gauss}}$

with $I_{\mathrm{qz}}$ being the average greyscale value of the "quartz" phase in the original FIB-SEM image, $A$ and $B$ being uniformly distributed random variables, $X$ and $Y$ being the pixel position in $X$ and $Y$ respectively, $\lambda$ being the wavelength of the sinusoid function, and Noise ${ }_{\text {Gauss }}$ being the varying levels of Gaussian noise (Fig. 2g).

These "combined textural and greyscale contrast" datasets provide an extreme challenge for traditional imageprocessing algorithms as the problem extends beyond a simple denoising challenge to extracting differential contrast on differing regions of the image, something which could lead to an extremely involved and challenging-to-identify image processing workflow. The FIB-SEM dataset was chosen as the base for the combined textural and greyscale contrast dataset suite (rather than another dataset natively exhibiting textural contrast) as it displayed significant structural complexity (with five distinct phases), and so posed a particular challenge for the algorithm. It also allows for the comparison of algorithmic performance between two suites of datasets reconstructed from the same original dataset to contain differing contrast mechanisms. 

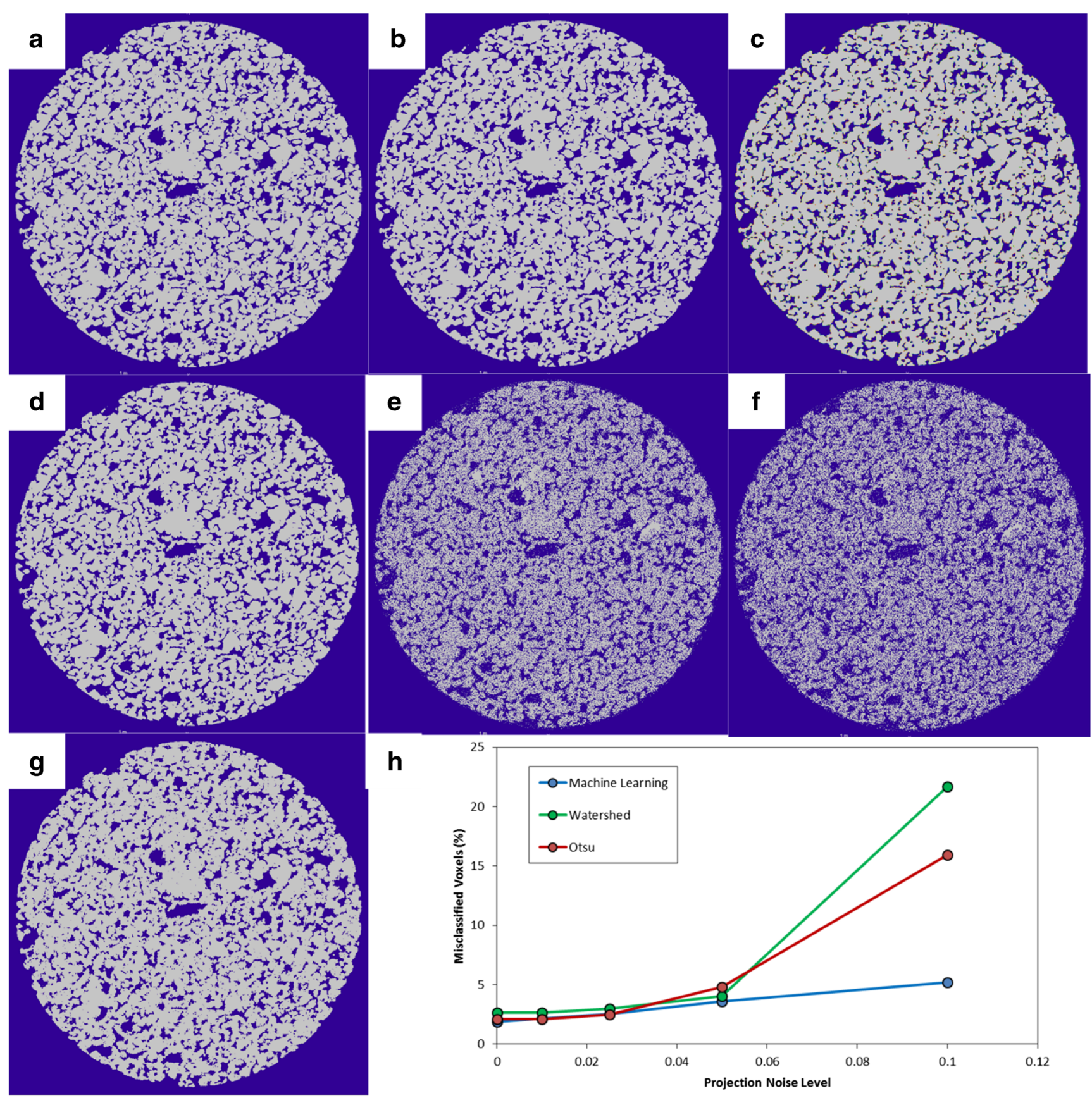

h

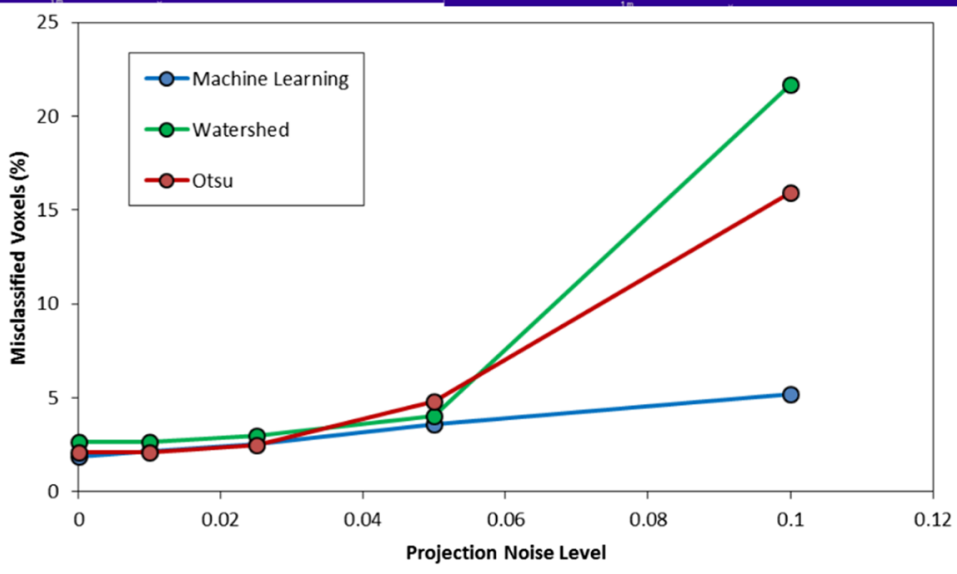

Fig. 3 Benchmarking XRM dataset of varying noise levels. a Original segmentation. $\mathbf{b}$ Segmentation using multi-Otsu techniques at noise $\sigma=0.01$. c Segmentation using seeded watershed algorithm at noise $\sigma=0.01$. d Segmentation using machine learning at noise $\sigma=0.01$.

\section{Benchmarking}

Each of the synthetic datasets was then segmented using three different techniques. First, multi-Otsu thresholding was used [35], an automated technique for finding a universal greyscale threshold by minimizing inter-class variance. Second, a watershed algorithm computed off a seed generated using a $2 \mathrm{D}$ histogram was performed e Segmentation using multiOtsu techniques at noise $\sigma=0.1$. f Segmentation using seeded watershed at noise $\sigma=0.1$. $\mathrm{g}$ Segmentation using machine learning algorithms at noise $\sigma=0.1$. h Relationship between number of misclassified voxels and projection noise level

[36]. Finally, a multiphase segmentation was performed, using the same workflow as described above. MultiOtsu thresholding and seeded watershed region growing were performed in ORS Dragonfly whereas the machine learning-based segmentation was performed in ZEISS Zen Intellesis. To analyze the fidelity with which each segmentation technique recovered the original segmented phase distribution, a logical XOR operation was performed 
between the original segmentation and the newly segmented images. The number of misclassified voxels belonging to a phase different to those in the original segmentation was then counted and expressed as a proportion of the original image. This was used as a quantitative metric for the performance of each image processing algorithm (Fig. 3). Frequently, image processing algorithms use pre- or postsegmentation de-noising filters to facilitate and improve the resulting segmentation. Pre-segmentation filters vary in complexity from simple mean pixel averaging through to complex non-linear edge preserving filters (e.g., the nonlocal means (NLM) filter [37, 38]), and post-segmentation filters include morphological filters such as image Opening, Closing, and Majority filtering. Their impact may seem to be an appropriate target for benchmarking efforts. In order to isolate the impact of specifically the segmentation step on the resulting data, however, only the segmentation technique was varied during benchmarking (no other image processing was performed). This represents variations in image quality of the image immediately prior to the segmentation step (independent of the rest of a potentially complex image processing workflow).

At low noise levels (Fig. 3b-d), all segmentation algorithms perform well with proportions of misclassified voxels $(<5 \%)$. At high noise levels (Fig. 3e-g), however, traditional techniques have a higher level of voxel misclassification. This can be seen in the segmented reconstructed image cross sections (Fig. 3e, f) by pore voxels in regions shown in Fig. 3a (the original segmentation) as grain and vice versa. The machine learning segmentation (Fig. 3g), however, performs well across the noise range. This can be seen in the plot of the noise level against the number of
Fig. 4 Re-segmentation of FIB-SEM datasets. a Original segmented data. b Segmentation using multi-Otsu techniques at noise $\sigma=10$. $\mathbf{c}$ Segmentation using seeded watershed algorithm at noise $\sigma=20$. $\mathbf{d}$ Segmentation using machine learning at noise $\sigma=10$. e Relationship between percent misclassified voxels and noise standard deviations
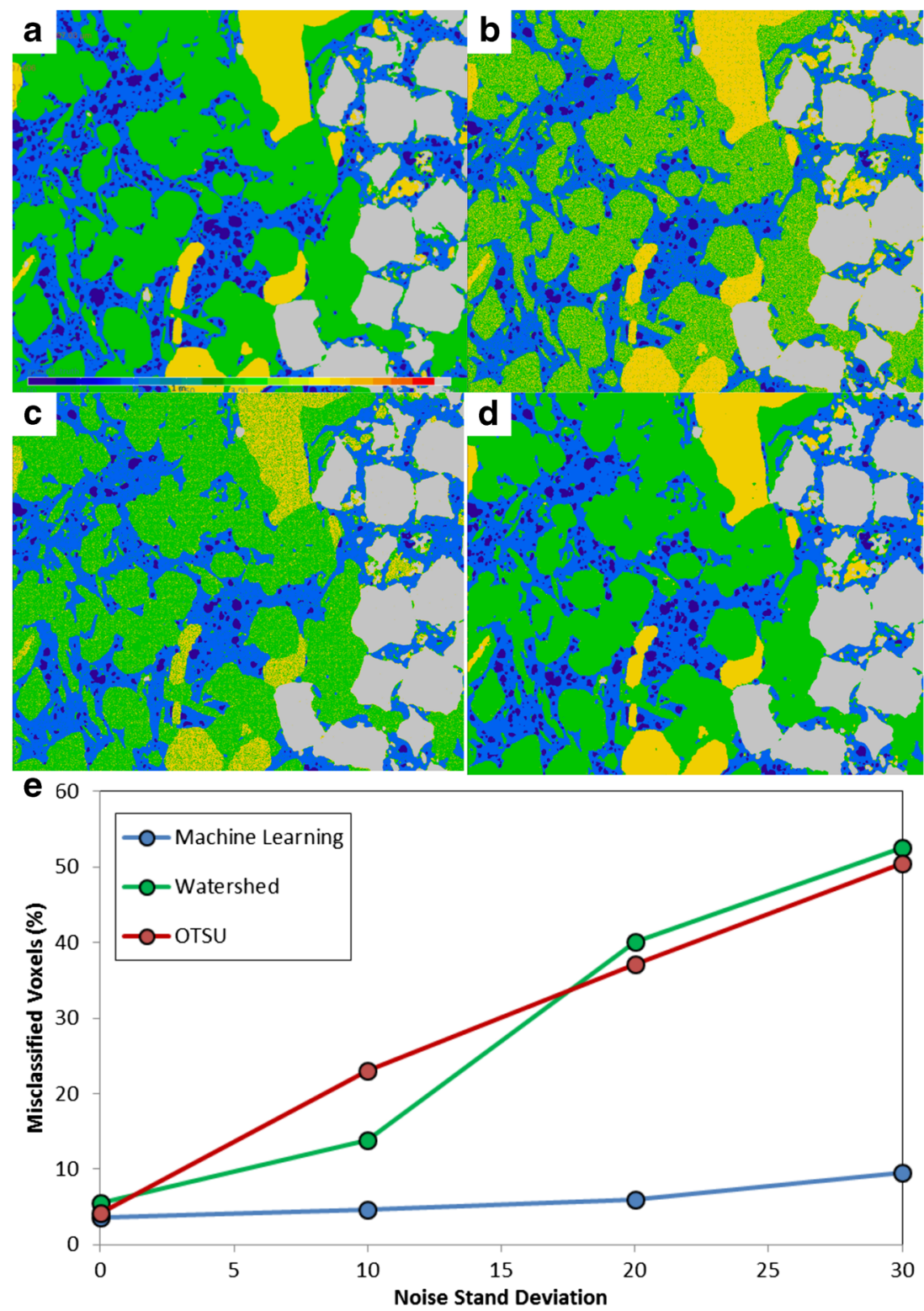
misclassified voxels-machine learning techniques retain a low level of misclassification (around 5\%), whereas the traditional technique misclassification rate increases significantly. One interesting feature of this analysis is that even when that dataset has no additional noise applied to the dataset, there is still a number of misclassified voxels (around $2.5 \%$ ). The filtered back projection process necessarily introduces some artefacts in the reconstructed data, which in turn introduces uncertainty in the location of the training regions. This is essentially reproducing the fact that, even in high-quality data, the human eye cannot interpret the data with $100 \%$ accuracy.

A similar analysis was performed for the "greyscale contrast" suite of synthetic FIB-SEM data (Fig. 4). All segmentation techniques produce good results (with low numbers of misclassified voxels) at low noise standard deviations; however, as the standard deviation of the noise increases, the level of greyscale misclassification increases dramatically. Interestingly, at intermediate noise in the FIB-SEM derived dataset, watershed outperforms simple Otsu thresholding. This may be because of the impact of partial volume artefacts (regions on phase interfaces where the greyscale value passes continuously through values associated with intermediate phases with average greyscale values between the average greyscale values of the two phases on either side of the interface) on voxel misclassifications at this noise level. We might expect region growing algorithms seeded in low-gradient areas to be less susceptible to the impact of this effect as it is the high-gradient regions that display these partial
Fig. 5 Re-segmentation of FIBSEM datasets exhibiting textural contrast. a Original segmented data. b Segmentation using multi-Otsu techniques at noise $\sigma=10$. c Segmentation using seeded watershed algorithm at noise $\sigma=10$. d Segmentation using machine learning at noise $\sigma=10$. e Relationship between percent misclassified voxels and noise standard deviations
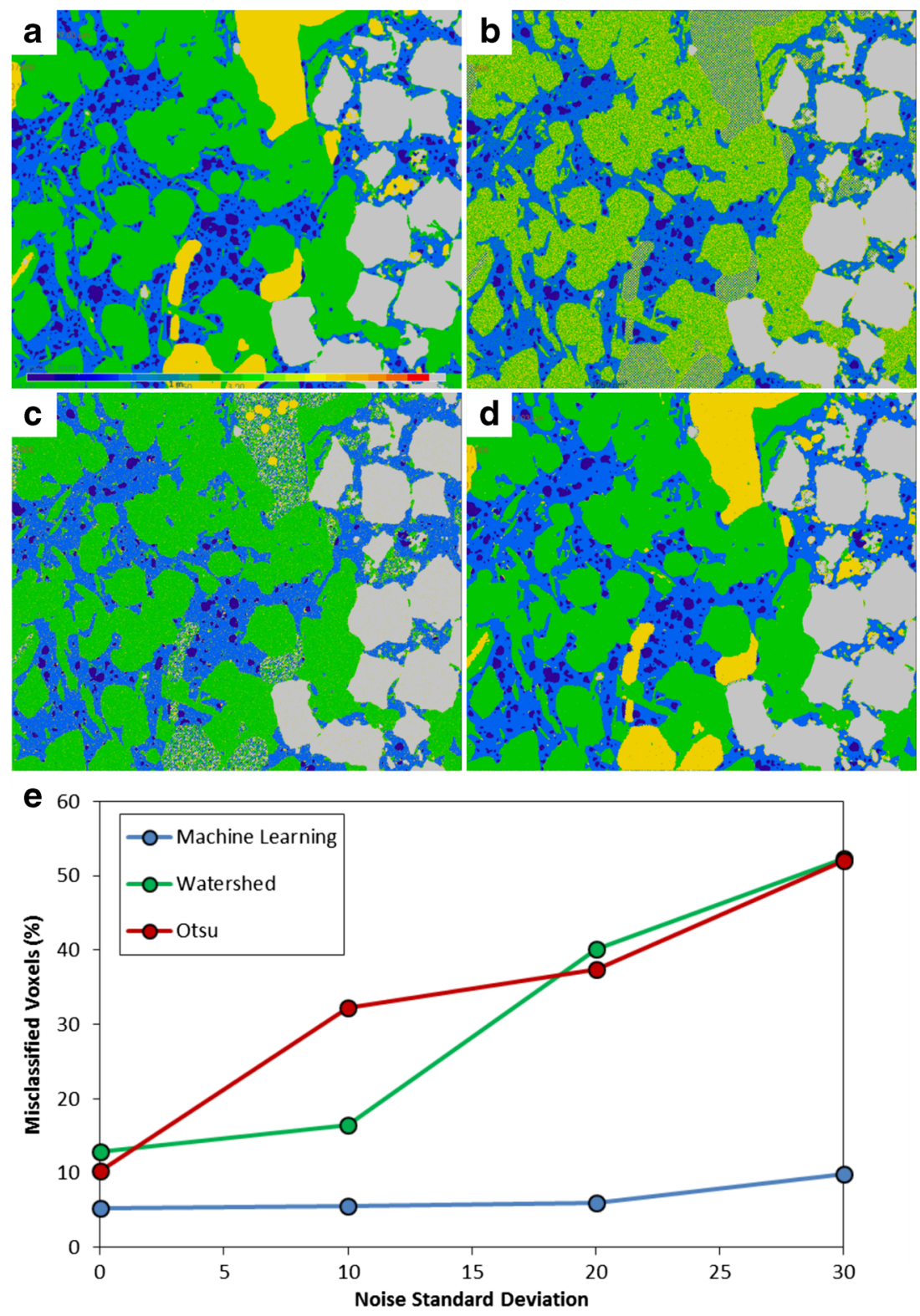
volume effects. At higher noise levels, however, both traditional algorithms perform equally poorly. The machine learning-based segmentation performs much better-even at the highest noise levels, it shows relatively low voxel misclassifications $(<10 \%)$.

The final suite of synthetic data to be analyzed were those where the data displayed both greyscale and textural contrast (with the textural contrast specifically displayed only in the region occupied by the "calcite" phase with the fourth highest greyscale in the original dataset (Fig. 5)). This textural contrast is a particularly interesting case as, frequently, when we visually inspect images, textural or morphological differences present the most striking differences between different phases. In the petroleum geosciences, there is particular interest in this as, frequently, mineralogical differences may be expressed by such variations in image texture or morphology.

The combined greyscale / textural contrast dataset shows broadly similar patterns to the greyscale contrast only dataset, but with some critical differences, particularly in the region exhibiting textural contrast. Similar to the greyscaleonly synthetic data, this textural contrast data shows relative performance improvements of seeded watershed techniques over multi-Otsu thresholding at intermediate noise datasets $(\sigma=10)$, but very similar (poor) performance at larger noise levels. Machine learning techniques perform well across all image noise levels. An interesting difference relative to the greyscale only contrast dataset is the significantly increased voxel misclassification at very low noise levels. An interesting difference, however, is the significantly increased voxel misclassification at very low noise levels. This is associated with misclassifications within the "calcite" region displaying textural contrast. Machine learning, however, gives a much lower level of voxel misclassification, similar to that in the original greyscale contrast suite of synthetic data. This is because traditional image processing techniques are sensitive only to a greyscale contrast-within the textural contrast region, they perform no better than simply randomly assigning voxel classifications. The machine learning-based segmentation, however, has features (such as Hessian and Gabor filters). These features are then used as components for a multivariant classification scheme. Regions exhibiting greyscale contrast (the phases corresponding to the pores, organics, and pyrite) are being classified using local and non-local greyscale features, and the regions distinguished by the textural contrast region (corresponding to the quartz and calcite in the original image) are being classified using a combination of textural and greyscale features. The relative capacity of the different segmentation to classify based on texture can be seen by examining the fraction of misclassified voxels within the textural contrast phase (Fig. 6).

Within this region, voxel misclassifications for traditional techniques are high even at low noise levels, and were above $50 \%$ across the noise range. Machine learning techniques, however, have feature vector components built into them that extract textural information and allow this information to be used for discrimination in classification. This allows misclassifications to be kept low $(<15 \%)$ across the noise range even within the textural contrast region.
Fig. 6 Voxel misclassifications within the textural contrast region

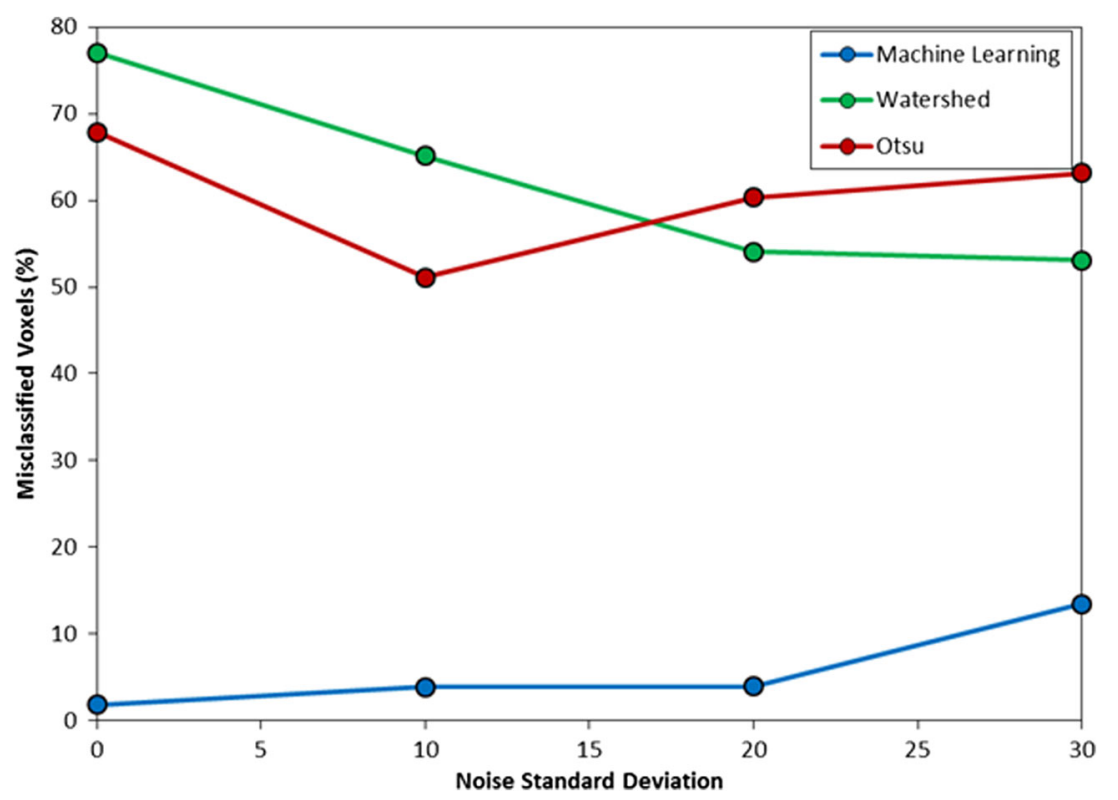




\section{Conclusions}

The relative performance of three segmentation techniques (multi-Otsu thresholding, seeded watershed region growing, and machine learning-based classification) were quantitatively assessed on three suites of synthetic data constructed from two different imaging modalities. The first suite of synthetic data was produced from an XRM dataset of a sandstone sample, to which variable levels of noise were introduced into the projection domain. This found that, while traditional techniques perform well at low noise levels, at high noise levels they fail to recover the original segmented geometry, with large numbers of misclassified voxels. Similar behavior was found for the second suite of synthetic data, constructed from a five-phase nanoscale FIB-SEM image of a shale sample exhibiting only greyscale contrast information, with traditional techniques performing well at low noise levels but poorly (with high rates of voxel misclassification) at higher noise levels. The final suite of synthetic data was constructed from the same FIBSEM shale image as the second suite of synthetic data but with one of the phases exhibiting textural (rather than greyscale) contrast. All traditional techniques completely failed to perform accurate segmentation within this textural contrast region, even at low noise levels; traditional techniques are completely insensitive to non-greyscale contrast. Overall, machine learning outperformed traditional techniques across a wide range of noise levels across multiple sample types, especially when trying to discriminate features based on texture.

The advent of machine learning technologies presents a unique opportunity in microstructural analysis. The increased noise tolerance of machine learning-based classification techniques has the potential for allowing the same analytical results to be performed with significantly noisier data, creating the opportunity for significantly reduced acquisition times without sacrificing analytical accuracy. This could allow for the examination of dynamic in situ experimentation at a higher temporal resolution. It also has the potential for automating industrial analytical workflows, particularly on repetitive samples. Machine learning-based classification could be trained on one dataset then applied across multiple samples to give repetitive, non-subjective results. The ability to classify based on features other than just local greyscale value, particularly the ability to classify based on textural information, has the potential of being transformative in our ability to extract information from images in the geosciences, ranging from 3D segmentation of mineralogical information to 3D segmentation of microfacies, to automated segmentation of mineralogy from transmitted light microscopy data. Beyond this, there are a host of different applications of machine learning techniques beyond the relatively simple challenge of pixel classification, including whole image classification, object detection, and defect recognition.

Acknowledgements I thank ZEISS microscopy, especially the ZEISS Zen Intellesis team, for the equipment and software access required for this work. I also thank Dr. Lori Hathon, Dr. Sreenivas Bhattiprolu and Dr. Lorenz Lechner for help and discussion.

Author contributions All work was performed by the sole author of this manuscript (Dr. Matthew Andrew).

Open Access This article is distributed under the terms of the Creative Commons Attribution 4.0 International License (http:// creativecommons.org/licenses/by/4.0/), which permits unrestricted use, distribution, and reproduction in any medium, provided you give appropriate credit to the original author(s) and the source, provide a link to the Creative Commons license, and indicate if changes were made.

\section{References}

1. Raeini, A.Q., Bijeljic, B., Blunt, M.J.: Numerical modelling of sub-pore scale events in two-phase flow through porous media. Transp. Porous Media 101, 191-213 (2014). https://doi.org/10. 1007/s11242-013-0239-6

2. Wildenschild, D., Sheppard, A.P.: X-ray imaging and analysis techniques for quantifying pore-scale structure and processes in subsurface porous medium systems. Adv. Water Resour. 51, 217 246 (2013). https://doi.org/10.1016/j.advwatres.2012.07.018

3. Blunt, M.J., Bijeljic, B., Dong, H., Gharbi, O., Iglauer, S., Mostaghimi, P., Paluszny, A., Pentland, C.: Pore-scale imaging and modelling. Adv. Water Resour. 51, 197-216 (2013). https://doi. org/10.1016/j.advwatres.2012.03.003

4. Cnudde, V., Boone, M.N.: High-resolution X-ray computed tomography in geosciences: a review of the current technology and applications. Earth-Science Rev. 123, 1-17 (2013). https://doi.org/10.1016/j.earscirev.2013.04.003

5. Iglauer, S., Paluszny, A., Pentland, C.H., Blunt, M.J.: Residual CO2 imaged with X-ray micro-tomography. Geophys. Res. Lett. 38, 1-6 (2011). https://doi.org/10.1029/2011GL049680

6. Andrew, M., Bijeljic, B., Blunt, M.J.: Pore-scale imaging of geological carbon dioxide storage under in situ conditions. Geophys. Res. Lett. 40, 3915-3918 (2013)

7. Herring, A.L., Andersson, L., Newell, D.L., Carey, J.W., Wildenschild, D.: Pore-scale observations of supercritical $\mathrm{CO} 2$ drainage in Bentheimer sandstone by synchrotron x-ray imaging. Int. J. Greenh. Gas Control 25, 93-101 (2014). https://doi.org/10.1016/j. ijggc.2014.04.003

8. Menke, H.P., Andrew, M.G., Blunt, M.J., Bijeljic, B.: Reservoir condition imaging of reactive transport in heterogeneous carbonates using fast synchrotron tomography-effect of initial pore structure and flow conditions. Chem. Geol. 428, 15-26 (2016). https://doi.org/10.1016/j.chemgeo.2016.02.030

9. Menke, H., Bijeljic, B., Andrew, M., Blunt, M.J.: Dynamic porescale imaging of reactive transport in heterogeneous carbonates at reservoir conditions. Energy Procedia 63, 5503-5511 (2014)

10. Armstrong, R.T., Porter, M.L., Wildenschild, D.: Linking porescale interfacial curvature to column-scale capillary pressure. Adv. Water Resour. 46, 55-62 (2012). https://doi.org/10.1016/j.advwat res.2012.05.009 
11. Andrew, M., Bijeljic, B., Blunt, M.J.: Pore-by-pore capillary pressure measurements using X-ray microtomography at reservoir conditions: curvature, snap-off, and remobilization of residual CO2. Water Resour. Res. 50, 8760-8774 (2014). https://doi.org/ 10.1002/2014WR015970

12. Andrew, M., Bijeljic, B., Blunt, M.J.: Pore-scale contact angle measurements at reservoir conditions using X-ray microtomography. Adv. Water Resour. 68, 24-31 (2014). https://doi.org/10.1016/ j.advwatres.2014.02.014

13. Scanziani, A., Singh, K., Blunt, M.J., Guadagnini, A.: Automatic method for estimation of in situ effective contact angle from X-ray micro tomography images of two-phase flow in porous media. J. Colloid Interface Sci. 496, 51-59 (2017). https://doi.org/10.1016/ j.jcis.2017.02.005

14. AlRatrout, A., Raeini, A.Q., Bijeljic, B., Blunt, M.J.: Automatic measurement of contact angle in pore-space images. Adv. Water Resour. 109, 158-169 (2017). https://doi.org/10.1016/j.advwatres. 2017.07.018

15. Aghaei, A., Piri, M.: Direct pore-to-core up-scaling of displacement processes: dynamic pore network modeling and experimentation. J. Hydrol. 522, 488-509 (2015). https://doi.org/10.1016/j. jhydrol.2015.01.004

16. Andrä, H., Combaret, N., Dvorkin, J., Glatt, E., Han, J., Kabel, M., Keehm, Y., Krzikalla, F., Lee, M., Madonna, C., Marsh, M., Mukerji, T., Saenger, E.H., Sain, R., Saxena, N., Ricker, S., Wiegmann, A., Zhan, X.: Digital rock physics benchmarks-part I: imaging and segmentation. Comput. Geosci. 50, 25-32 (2013). https://doi.org/10.1016/j.cageo.2012.09.005

17. Andrä, H., Combaret, N., Dvorkin, J., Glatt, E., Han, J., Kabel, M., Keehm, Y., Krzikalla, F., Lee, M., Madonna, C., Marsh, M., Mukerji, T., Saenger, E.H., Sain, R., Saxena, N., Ricker, S., Wiegmann, A., Zhan, X.: Digital rock physics benchmarks-part II: computing effective properties. Comput. Geosci. 50, 33-43 (2013). https://doi.org/10.1016/j.cageo.2012.09.008

18. Saxena, N., Hofmann, R., Alpak, F.O., Dietderich, J., Hunter, S., Day-Stirrat, R.J.: Effect of image segmentation \& voxel size on micro-CT computed effective transport \& elastic properties. Mar. Pet. Geol. 86, 972-990 (2017). https://doi.org/10.1016/j.marpetge o.2017.07.004

19. Schlüter, S., Sheppard, A., Brown, K., Wildenschild, D.: Image processing of multiphase images obtained via X-ray microtomography: a review. Water Resour. Res. 50, 3615-3639 (2014). https://doi.org/10.1002/2014WR015256

20. Chauhan, S., Rühaak, W., Khan, F., Enzmann, F., Mielke, P., Kersten, M., Sass, I.: Processing of rock core microtomography images: using seven different machine learning algorithms. Comput. Geosci. 86, 120-128 (2016). https://doi.org/10.1016/j.cageo. 2015.10.013

21. Koebernick, N., Daly, K.R., Keyes, S.D., George, T.S., Brown, L.K., Raffan, A., Cooper, L.J., Naveed, M., Bengough, A.G., Sinclair, I., Hallett, P.D., Roose, T.: High-resolution synchrotron imaging shows that root hairs influence rhizosphere soil structure formation. New Phytol 216, 124-135 (2017). https://doi.org/10.1111/nph.14705

22. Schweizer, S.A., Hoeschen, C., Schlüter, S., Kögel-Knabner, I., Mueller, C.W.: Rapid soil formation after glacial retreat shaped by spatial patterns of organic matter accrual in microaggregates. Glob. Chang. Biol. 24, 1637-1650 (2018). https://doi.org/10.1111/ gcb. 14014

23. Øren, P.E., Bakke, S., Arntzen, O.J.: Extending predictive capabilities to network models. SPE J. 3, 324-336 (1998)
24. Øren, P.E., Bakke, S.: Process based reconstruction of sandstones and prediction of transport properties. Transp. Porous Media 46, 311-343 (2002). https://doi.org/10.1023/A:1015031122338

25. Milliken, K.L., Rudnicki, M., Awwiller, D.N., Zhang, T.: Organic matter-hosted pore system, Marcellus Formation (Devonian), Pennsylvania. Am. Assoc. Pet. Geol. Bull. 97, 177-200 (2013). https://doi.org/10.1306/07231212O48

26. Loucks, R.G., Reed, R.M., Ruppel, S.C., Hammes, U.: Spectrum of pore types and networks in mudrocks and a descriptive classification for matrix-related mudrock pores. Am. Assoc. Pet. Geol. Bull. 96, 1071-1098 (2012). https://doi.org/10.1306/081711 11061

27. Jiao, K., Yao, S., Liu, C., Gao, Y., Wu, H., Li, M., Tang, Z.: The characterization and quantitative analysis of nanopores in unconventional gas reservoirs utilizing FESEM-FIB and image processing: an example from the lower Silurian Longmaxi Shale, upper Yangtze region. China. Int. J. Coal Geol. 128-129, 1-11 (2014). https://doi.org/10.1016/j.coal.2014.03.004

28. Wang, P., Jiang, Z., Ji, W., Zhang, C., Yuan, Y., Chen, L., Yin, L.: Heterogeneity of intergranular, intraparticle and organic pores in Longmaxi shale in Sichuan Basin, South China: evidence from SEM digital images and fractal and multifractal geometries. Mar. Pet. Geol. 72, 122-138 (2016). https://doi.org/10.1016/j.marpetg eo.2016.01.020

29. Ju, Y., Gong, W., Chang, C., Xie, H., Xie, L., Liu, P.: Threedimensional characterisation of multi-scale structures of the Silurian Longmaxi shale using focused ion beam-scanning electron microscopy and reconstruction technology. J. Nat. Gas Sci. Eng. 46, 26-37 (2017). https://doi.org/10.1016/j.jngse.2017.07.015

30. Andrew, M., Bijeljic, B., Blunt, M.J.: Pore-scale imaging of trapped supercritical carbon dioxide in sandstones and carbonates. Int. J. Greenh. Gas Control 22, 1-14 (2014). https://doi.org/10. 1016/j.ijggc.2013.12.018

31. Feldkamp, L.A., Davis, L.C., Kress, J.W.: Practical cone-beam algorithm. J. Opt. Soc. Am. A 1, 612 (1984). https://doi.org/10. 1364/JOSAA.1.000612

32. Andrew, M.G., Bhattiprolu, S., Butnaru, D., Correa, J.: The usage of modern data science in segmentation and classification: machine learning and microscopy. Microsc. Microanal. 23, 156157 (2017)

33. Breiman, L.: Random forests. Mach. Learn. 45, 5-32 (2001). https://doi.org/10.1023/A:1010933404324

34. Lai, P., Moulton, K., Krevor, S.: Pore-scale heterogeneity in the mineral distribution and reactive surface area of porous rocks. Chem. Geol. 411, 260-273 (2015). https://doi.org/10.1016/j.chem geo.2015.07.010

35. Otsu, N.: A threshold selection method from gray-level histograms. IEEE Trans. Syst. Man. Cybern. 9, 62-66 (1979). https://doi.org/10.1109/TSMC.1979.4310076

36. Jones, A.C., Arns, C.H., Sheppard, A.P., Hutmacher, D.W., Milthorpe, B.K., Knackstedt, M.A.: Assessment of bone ingrowth into porous biomaterials using MICRO-CT. Biomaterials 28, 2491-2504 (2007). https://doi.org/10.1016/j.biomaterials.2007. 01.046

37. Buades, A., Coll, B., Morel, J.-M.: Nonlocal image and movie denoising. Int. J. Comput. Vis. 76, 123-139 (2008). https://doi.org/ 10.1007/s11263-007-0052-1

38. Buades, A., Coll, B., Morel, J.-M.: A non-local algorithm for image denoising. Comput. Vis. Pattern Recognit. https://doi.org/ 10.1109/CVPR.2005.38 (2005) 